HM Herbert ${ }^{1}$, K Jordan² and DW Flanagan

\section{Is screening with digital imaging using one retinal view adequate?}

threshold for slit-lamp biomicroscopy if image quality is poor.

Eye (2003) 17, 497-500. doi:10.1038/

sj.eye. 6700409

Purpose To compare the detection of diabetic retinopathy from digital images with slit-lamp biomicroscopy, and to determine whether British Diabetic Association (BDA) screening criteria are attained ( $>80 \%$ sensitivity, $>95 \%$ specificity, $<5 \%$ technical failure).

Methods Diabetics referred for screening were studied in a prospective fashion. A single $45^{\circ}$ fundus image was obtained using the nonmydriatic digital camera. Each patient subsequently underwent slit-lamp biomicroscopy and diabetic retinopathy grading by a consultant ophthalmologist. Diabetic retinopathy and maculopathy was graded according to the Early Treatment of Diabetic Retinopathy Study.

Results A total of 145 patients (288 eyes) were identified for screening. Of these, $26 \%$ of eyes had diabetic retinopathy, and eight eyes (3\%) had sight-threatening diabetic retinopathy requiring treatment. The sensitivity for detection of any diabetic retinopathy was $38 \%$ and the specificity $95 \%$. There was a $4 \%$ technical failure rate. There were $42 / 288$ false negatives and 10/288 false positives. Of the 42 false negatives, 18 represented diabetic maculopathy, 20 represented peripheral diabetic retinopathy and four eyes had both macular and peripheral changes. Three eyes in the false-negative group (1\% of total eyes) had sight-threatening retinopathy. There was good concordance between the two consultants $(79 \%$ agreement on slit-lamp biomicroscopy and $84 \%$ on digital image interpretation).

Conclusion The specificity value and technical failure rate compare favourably with BDA guidelines. The low sensitivity for detection of any retinopathy reflects failure to detect minimal maculopathy and retinopathy outside the $45^{\circ}$ image. This could be improved by an additional nasal image and careful evaluation of macular images with a low
Keywords: diabetic retinopathy; digital; screening

\section{Introduction}

Diabetic retinopathy is an important public health concern and is the most common cause of blindness in our working population. ${ }^{1,2}$ Diabetic retinopathy is seen in most type I diabetics and $60 \%$ of type II diabetics 20 years after diagnosis. ${ }^{3}$ Approximately $2 \%$ of the UK population have diabetes, of whom 200000 have type I (insulin dependent) and more than 1 million have type II (non-insulin dependent). ${ }^{4}$

The St Vincent declaration (1990) included among its objectives the reduction of incidence of blindness caused by diabetic retinopathy within 10 years.

Diabetic retinopathy fulfils the WHO criteria for a screening programme. Screening for diabetic retinopathy is a cost-effective exercise because early detection and treatment has been shown to prevent visual impairment, and the costs in terms of managing the effects of severe visual impairment/blindness can be effectively reduced. ${ }^{5}$ The quality of life benefits due to early detection of sight-threatening retinopathy and early treatment are significant.

Screening should be combined with good diabetic control. The Diabetes Control and Complications Trial highlighted the importance in insulin-dependent diabetes mellitus (IDDM) of good diabetic control in reducing the risk of progression of retinopathy. ${ }^{6}$ Similarly, the UKPDS demonstrated that good control of both blood pressure and diabetes in type II diabetics significantly reduced the risk of microvascular end points related to diabetes including retinopathy. ${ }^{7}$
${ }^{1}$ Department of Ophthalmology Hinchingbrooke Hospital Hinchingbrooke Park Huntingdon Cambridgeshire, UK

${ }^{2}$ Department of Ophthalmology West Suffolk Hospital Bury St Edmunds Suffolk, UK

Correspondence: HM Herbert St Paul's Eye Unit Royal Liverpool University Hospital

Prescott Street Liverpool, L7 8XP, UK Tel: + 441517062000 E-mail: helen.herbert@ ntlworld.com

Received: 19 December 2001

Accepted in revised form: 7 October 2002

This paper was presented at Royal College of Ophthalmologists Annual Congress, May 2001 
The benefits of laser photocoagulation are well known. The Diabetic Retinopathy Study confirmed the beneficial effects of laser photocoagulation in preventing severe visual loss in patients with proliferative diabetic retinopathy (PDR) and high-risk characteristics. ${ }^{8}$ The Early Treatment Diabetic Retinopathy Study (ETDRS) showed that focal or grid photocoagulation of clinically significant macular oedema substantially reduced the risk of moderate visual loss. ${ }^{9}$

Screening studies have shown that up to $10 \%$ of diabetics have sight-threatening retinopathy. ${ }^{10,11} \mathrm{Up}$ to $40 \%$ of type II diabetics have some retinopathy at diagnosis.

Early diabetic retinopathy can be detected by fundal examination prior to the development of symptoms.

The British Diabetic Association (BDA) have proposed criteria for effective screening. These standards have been used to assess and compare screening programmes. Sensitivity values of $>80 \%$ and specificity values of $>95 \%$ combined with a technical failure rate of $<5 \%$ are considered the standard to aim for in retinopathy detection. $^{12}$

There is agreement nationally about the need for diabetic retinopathy screening programmes; however, there is no universally agreed method for screening.

The Liverpool Diabetic Eye Study compared direct ophthalmoscopy through dilated pupils by an experienced ophthalmologist which had a sensitivity of $65 \%$, with fundus photography with mydriasis, three overlapping $45^{\circ}$ fields and a trained grader which had a sensitivity of $89 \% .^{13}$

A review of published literature analysed 22 cohort studies of diabetic retinopathy screening using retinal photography, direct ophthalmoscopy or both. They demonstrated considerable variation in both sensitivity and specificity levels achieved by different studies. The authors concluded that mydriatic retinal photography provides the most effective screening and monitoring test for detection of sight-threatening diabetic retinopathy. ${ }^{6}$ In general, the sensitivity and specificity levels were higher in studies where retinal photography was the method of choice.

Instant digital images have been shown to be superior to standard polaroid photography not only in terms of detecting retinopathy, but also in terms of image storage, accessibility and the potential for image enhancement and analysis. ${ }^{15}$

George et al ${ }^{16}$ compared digital imaging with $35 \mathrm{~mm}$ colour transparencies and showed that there is goodexcellent agreement between the two systems. There was exact agreement in $93.3 \%$ of images; however, $5.3 \%$ were undergraded and three cases of sight-threatening diabetic retinopathy (STDR) were graded as nonsightthreatening. They noted that the lesions most difficult to diagnose on the digital image were intraretinal microvascular abnormalities. They also found that drusen could be overgraded as hard exudates after software manipulation to improve resolution, because of an increase in contrast.

In a further study, George et $a l^{16}$ showed that software manipulation of digitised retinal images can further improve the level of agreement in grading as compared with $35 \mathrm{~mm}$ transparencies.

There is a need for efficiency in screening programmes. The question remains as to how many fundal images are required to identify diabetic retinopathy adequately.

\section{Aims}

In Hinchingbrooke Hospital, diabetic screening for retinopathy has until recently been carried out using standard fundus photography. The department acquired a digital camera to replace previous methods of screening. We set up a prospective study to evaluate the screening service provided by the department and determine which aspects could be improved.

Our specific aims were as follows:

1. To determine the effectiveness of screening using the digital camera and a single $45^{\circ}$ retinal image.

2. To compare the digital camera with the 'gold standard', that is, slit-lamp biomicroscopy.

3. To see whether screening using the digital camera meets the criteria proposed by the BDA.

\section{Materials and methods}

A prospective study was set up. Statistical advice regarding sample size recommended that at least 143 patients (286 eyes) were screened for diabetic retinopathy. All patients due to attend screening were included in the study. Ethical approval was not sought, as patients were due for screening appointments.

Their general practitioner (GP), the diabetic liaison sister or the diabetic physician referred the patients for screening. Patients were advised on arrival that they would be photographed and then assessed by a consultant ophthalmologist to screen for diabetic retinopathy.

Patient details such as age, type and duration of diabetes were recorded on a standard screening letter for their GP. This was updated and included definitions of diabetic retinopathy were included in keeping with the ETDRS.

Snellen visual acuity (best corrected) was measured by a nurse. This was then rechecked by an ophthalmologist.

A digital nonmydriatic camera was used for screening (TRC NW5-S model, $800 \times 600$ Sony 3-Chip). A single $45^{\circ}$ 
image was obtained centred on the macula. Digital images were obtained (without mydriasis) by a member of the nursing staff unless images were difficult to obtain.

If images were inadequate $G$ Tropicamide $0.5 \%$ was instilled and imaging repeated. The aim was to obtain good-quality images for evaluation. Patients were excluded from the study if images were blurred ('technical failures') despite mydriasis.

Slit-lamp biomicroscopy was performed by a retinal specialist, and diabetic retinopathy graded. A second independent retinal specialist assessed the digital images, and graded the retinopathy. The two independent retinal specialists were consultants with extensive experience of screening for and treating diabetic retinopathy. Each consultant was 'blinded' as to the other's findings to reduce the potential for bias in grading.

Diabetic retinopathy was defined in line with ETDRS and the DRS definitions. The grades of retinopathy were modified for simplification (Tables 1 and 2).

The presence of diabetic maculopathy was identified within two disc diameters of the fovea. Maculopathy was defined as 'minimal' or 'treatable'. Treatable maculopathy included patients where clinically significant macular oedema was detected. Eyes where maculopathy was present but not sight-threatening were graded as 'minimal maculopathy'.

Retinopathy was graded as mild, moderate and severe non-proliferative diabetic retinopathy (NPDR), or (PDR).

A subgroup of 18 patients were examined by both retinal specialists and the digital images were regraded after a 3-month interval by both specialists. This subgroup was used to assess the concordance of the

Table 1 Grading of diabetic retinopathy

1. No retinopathy

2. Mild NPDR (at least one microaneurysm)

3. Moderate NPDR (extensive intraretinal haemorrhages +/or microaneurysms, +/or cotton wool spots, venous beading or IRMA, not as severe as 'severe NPDR'.)

4. Severe NPDR (cotton wool spots, venous beading, IRMA. All present in at least two quadrants or two of them present in at least two quadrants with MAs and intraretinal haemorrhages in all four quadrants or IRMA in all four quadrants, (severe in at least one). No PDR.)

5. PDR

Table 2 Grading of maculopathy

\begin{tabular}{ll}
\hline A & $\begin{array}{l}\text { No maculopathy } \\
\text { Minimal maculopathy (maculopathy with no clinically } \\
\text { significant macular oedema, CSMO) }\end{array}$ \\
B & Treatable maculopathy (with CSMO)
\end{tabular}

If minimal maculopathy was present in a patient with moderate NPDR, they were graded as $3 \mathrm{a}$. A grade of $4 \mathrm{~b}$ defined severe NPDR with CSMO. retinal specialists in grading the fundal findings by both slit-lamp biomicroscopy and digital imaging.

\section{Results}

A total of 301 eyes were identified for screening. Blurred images (technical failures) were noted in $4 \%$ of eyes screened and were excluded from the study leaving 288 eyes (145 patients) suitable for the study.

Of these, $27 \%$ had type I diabetes (39/145) and $73 \%$ of patients had type II diabetes (106/145). The majority of the type II diabetics (69\%) were tablet-controlled, 20\% were diet-controlled and $9 \%$ were insulin-requiring ( $2 \%$ not recorded).

In all, 288 eyes were suitable for screening using the digital camera, $26 \%$ of eyes had diabetic retinopathy and eight eyes $(3 \%)$ had STDR requiring treatment.

The sensitivity for detection of any diabetic retinopathy was 38\% and the specificity $95 \%$ (Table 3 ).

There were $42 / 288$ (15\%) false negatives. Of these 42 eyes, 18 had diabetic maculopathy, 20 eyes had peripheral diabetic retinopathy and four eyes had both maculopathy and peripheral changes. Three eyes in the false-negative group ( $1 \%$ of total eyes) had sightthreatening retinopathy.

There were 10/288 false positives. Of these, six eyes had diabetic maculopathy and four eyes had peripheral changes.

The two consultants concordance with grading was $79 \%$ on slit-lamp biomicroscopy and $84 \%$ on digital image interpretation.

\section{Discussion}

The population of patients in this study represents a 'normal' screening population. This is reflected in the incidence of any diabetic retinopathy that was $26 \%$. The incidence of sight-threatening retinopathy is low and therefore sensitivity and specificity figures for any retinopathy are calculated.

This study shows that care needs to be taken when screening patients using digital imaging. Clear, goodquality images are essential. These can be obtained without mydriasis in the majority of patients; however,

Table 3 Results of screening

\begin{tabular}{lcc}
\hline & $\begin{array}{c}\text { DR detected on } \\
\text { slit lamp }\end{array}$ & $\begin{array}{c}\text { No DR on } \\
\text { slit lamp }\end{array}$ \\
\hline DR detected on digital image & 26 & 10 \\
No DR on digital image & 42 & 210 \\
\hline
\end{tabular}

DR: diabetic retinopathy; sensitivity: $26 / 68=38.2 \%$;

specificity: $210 / 220=95.5 \%$; technical failure: $13 / 301=4.3 \%$.

Total eyes $=301 ; 13$ eyes excluded because of blurred digital images. 
mydriatics should be used if there is any doubt. The technical failure rate in this group of patients (4\%) is in line with BDA guidelines. Those eyes with blurred images despite mydriasis were excluded mainly because of media opacity such as cataract, and other reasons such as poor compliance or difficult head position.

Our sensitivity in this study (38.2\%) is low and not in line with BDA guidelines of $80 \%$. Sensitivity values of comparable studies range between 27 and $100 \%{ }^{6}$

The sensitivity level for this screening method could be improved by taking care in interpreting slightly blurred images. If there is any doubt about the image quality, the patient should be examined clinically. This is particularly important in patients with early cataract and slightly reduced visual acuities.

One image for screening is not adequate. The use of a second image centred on the optic disc should also increase sensitivity values. This second image would enable the nasal watershed areas to be visualised. Since these areas frequently show early ischaemia, the second image should improve the detection of peripheral changes, thus reducing this proportion of the falsenegative group.

Particular care should be directed towards detecting maculopathy. In this study, a significant proportion of the false-negative group (18/42) had diabetic maculopathy that was not detected by digital image analysis, despite image enhancement.

The digital images were stored using Joint Photographic Experts Group (JPEG) software that compresses the image with loss of quality, in this system estimated at $10 \%$. This may be a factor contributing to the low sensitivity value as was recently demonstrated. ${ }^{17}$

The specificity and technical failure rates compare favourably with BDA guidelines.

As a result of this study, we are re-evaluating the screening protocol using two images as described above. All patients with blurred images are still examined using slit-lamp biomicroscopy.

\section{Conclusion}

Diabetic retinopathy has been screened using a variety of methods. The digital camera produces an instant image, which can be enhanced during analysis providing facilitated screening of patients. Image capture is possible without mydriasis in the majority of patients. This reduces the time taken for image capture, thus improving the overall efficiency of the clinic.

This is an effective method of screening and has recently been endorsed by the National Screening Committee Review of diabetic retinopathy screening, ${ }^{18}$ however, care is needed to ensure high levels of sensitivity and specificity are achieved. We have shown that a single digital fundal image is insufficient for screening purposes. We have also shown that regular appraisal of local screening methods is essential if we are to screen effectively and achieve levels of sensitivity and specificity as set out by the BDA consistently.

Proprietary interest: None

\section{References}

1 Evans J, Rooney C, Ashwood F, Dattani N, Wormald R. Blindness and partial sight in England and Wales: April 1990-March 1991. Health Trends 1996; 28(1): 5-12.

2 Ghafour IM, Allan D, Foulds WS. Common causes of blindness and visual handicap in the West of Scotland. Br J Ophthalmol 1983; 67: 209-213.

3 Klein R, Klein BE, Moss SE, Davis MD, DeMets DL. The Wisconsin epidemiological study of diabetic retinopathy. III. Prevalence and risk of diabetic retinopathy when age at diagnosis is 30 or more years. Arch Ophthalmol 1984; 527-532.

4 Calman K. The Annual Report of the Chief Medical Officer of the Department of Health for the Year 1997. London: The Stationary Office.

5 Javitt JC, Aiello LP. Cost effectiveness of detecting and treating diabetic retinopathy. Ann Intern Med 1996; 124: 164-169.

6 Hutchinson A, McIntosh A, Peters J, O'Keefe C, Khunti K, Baker R et al. Diab Med 2000; 495-506.

7 Bagga P, Verma D, Walton C, Masson EA, Hepburn DA. Diab Med 1998; 15: 780-782.

8 Diabetic Retinopathy Study Research Group. Diabetic Retinopathy Study Report No. 8. Ophthalmology 1981; 88: 583-600.

9 Early Treatment of Diabetic Retinopathy Study Research Group. Treatment techniques \& clinical guidelines for photocoagulation of diabetic macular oedema: ETDRS Report No. 2. Ophthalmology 1987; 94: 761-774.

10 Backlund LB, Algvere PV, Rosenqvist U. Early detection of diabetic retinopathy by a mobile retinal photography service in partnership with primary health care teams. Diab Med 1998; 15(Suppl 3): S32-S37.

11 Kerr D, Cavan DA, Jennings B, Dunnington C, Gold D, Crick $\mathrm{M}$. Beyond retinal screening: digital imaging in the assessment and follow up of patients with diabetic retinopathy. Diab Med 1998; 15: 878-882.

12 British Diabetic Association. Retinal photographic screening for diabetic eye disease. A British Diabetic Association Report, London, 1997.

13 Harding SP, Broadbent DM, Neoh C, White MC, Vora J. BMJ 1995; 311: 1131-1135.

14 Ryder REJ, Kong N, Bates AS, Sim J, Welch J, Kritzinger EE. Diab Med 1998; 15: 254-258.

15 George LD, Halliwell M, Hill R, Aldington SJ, Lusty J, Dunstan F. A comparison of digital retinal images and $35 \mathrm{~mm}$ colour transparencies in detecting and grading diabetic retinopathy. Diab Med 1998; 15: 250-253.

16 George LD, Lusty J, Owens DR, Ollerton RL. Br J Ophthalmol 1999; 83: 911-913.

17 Newsom RSB, Clover A, Costen MTJ, Sadler J, Newton J, Luff AJ et al. B J Ophthalmol 2001; 85: 799-802.

18 Gillow JT, Muir Gray JA. Eye 2001; 15: 1-2. 